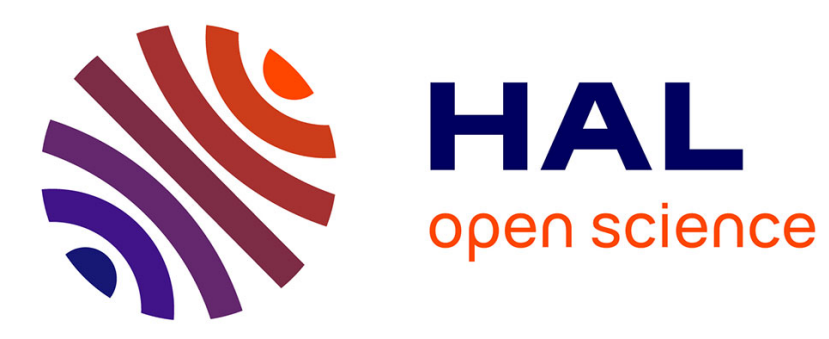

\title{
Interactive Scheduling Decision Support System for ore extraction at a phosphate mine
}

Najoua Alaoui, Ahlam Azzamouri, Selwa Elfirdoussi, Pierre Fenies, Vincent

Giard

\section{- To cite this version:}

Najoua Alaoui, Ahlam Azzamouri, Selwa Elfirdoussi, Pierre Fenies, Vincent Giard. Interactive Scheduling Decision Support System for ore extraction at a phosphate mine. 2019 International Conference on Industrial Engineering and Systems Management (IESM), Sep 2019, Shanghai, China. pp.1-6, 10.1109/IESM45758.2019.8948146 . hal-02484594

\section{HAL Id: hal-02484594 \\ https://hal.science/hal-02484594}

Submitted on 19 Feb 2020

HAL is a multi-disciplinary open access archive for the deposit and dissemination of scientific research documents, whether they are published or not. The documents may come from teaching and research institutions in France or abroad, or from public or private research centers.
L'archive ouverte pluridisciplinaire HAL, est destinée au dépôt et à la diffusion de documents scientifiques de niveau recherche, publiés ou non, émanant des établissements d'enseignement et de recherche français ou étrangers, des laboratoires publics ou privés. 


\section{Interactive Scheduling Decision Support System for ore extraction at a phosphate mine}

\author{
NAJOUA ALAOUI ${ }^{1}$ \\ ${ }^{l}$ EMINES, UNIVERSITY \\ MOHAMMED VI POLYTECHNIC \\ 43150 Ben Guerir, Morocco \\ Najoua.alaoui@emines.um6p.ma \\ PIERRE FENIES ${ }^{1,2}$ \\ ${ }^{l}$ EMINES, UNIVERSITY MOHAMMED VI \\ POLYTECHNIC \\ 43150 Ben Guerir, Morocco \\ ${ }^{2}$ PANTHEON-ASSAS, PARIS II UNIVERSITY \\ 75006 Paris, France \\ Pierre.Fenies@emines.um6p.ma
}

\begin{abstract}
- as open-pit mines consist in the accumulation of ore layers of different characteristics, their extraction process results from the iteration of a sequence of elementary operations performed on parcels by specialized or multipurpose machines. Medium-term extraction programming consists in deciding the assignment of the available machines over time. This problem is addressed through Discrete Event Simulation implemented in a phosphate mine, taking into account all the extraction constraints to drive the extraction process. This combines an effectiveness criterion (degree of urgency of obtaining certain ore qualities) and an efficiency criterion (machine displacements). While the parameterization used to control simulation quickly produces many technically feasible scenarios, making the final optimal choice is extremely difficult, particularly as one needs to take into account time considerations in extracted ore progressive provisioning and machine use. Our paper describes the bases of a visual decision-support system (DSS), currently under development, which enables the processing of results to compare multiple scenarios and adjust parameterization in order to come up with a good solution. This web-based DSS is coupled to the simulator via a relational database.
\end{abstract}

Keywords - Scheduling, Open-pit mine, Decision Support System (DSS), Graphical user interface.

\section{INTRODUCTION}

OCP SA is phosphate ore extraction and fertilizer production world leader. The tactical decisions that need to be made by OCP in connection with scheduling are related to ore extraction and depend on two criteria as explained in [1]: the choice of parcel to be extracted and the allocation of the machines to perform the operations. The aim of scheduling is to meet known or forecast demand by extracting specific deposit layers. The deposit contains $\mathrm{P}$ panels and each panel is made up of Np parcels. It is worth noting that OCP parcels are $4000 \mathrm{~m} 2$ rectangles that contain alternate phosphate and sterile layers. The solution to our scheduling decision problem must be able to i) identify the parcels to be extracted to obtain a specific layer of required Source Quality (SQ) ore and ii) allocate the extraction machines. In fact, the extracted SQs

\author{
SELWA ELFIRDOUSSI ${ }^{1}$ \\ ${ }^{l}$ EMINES, UNIVERSITY \\ MOHAMMED VI POLYTECHNIC \\ 43150 Ben Guerir, Morocco \\ Selwa.Elfirdoussi@emines.um6p.ma
}

\author{
VINCENT GIARD ${ }^{1,3}$ \\ ${ }^{1}$ EMINES, UNIVERSITY MOHAMMED VI \\ POLYTECHNIC \\ 43150 Ben Guerir, Morocco \\ ${ }^{3}$ PARIS-DAUPHINE, PSL RESEARCH UNIVERSITY \\ 75016, Paris, France \\ Vincent.Giard@emines.um6p.ma
}

correspond to the ore required in both nature and volume terms in order to produce and meet future demand for Merchantable Qualities (MQ) through a blending production process. Due to its size, the mining extraction mathematical programming model is hardly workable. We therefore designed a Discrete Event Simulation (DES) model with the aim of generating multiple feasible schedule scenarios [1]. Our model integrates a large number of parameters (mainly the prioritization of phosphate layer indexes, distance coefficients and layer accessibility as well as the technical specifications to allocate machines to their respective work including constraints associated with multipurpose machines, the minimum number of contiguous parcels that a machine must complete before moving to another location, etc.). All these parameters enable the user to adjust simulation behavior and the number of generated scenarios. Nevertheless, it is at best difficult, if not impossible, to come up with a compound indicator to evaluate overall scheduling option performance. Accordingly, we chose to compare the physical consequences of the different scenarios generated, particularly with respect to their overall efficiency.

Indeed, it is not enough for the simulation to be technically coherent for the scenarios to be valid. This led us to design a test to exclude scenarios leading to extract SQs unfit to meet global MQ demand. This rejection test involves a set of blending problems using the same composition constraints that are defined over several planning horizons $(1,3,6,9$ and 12 months) and relying on standard LP blending formulation [2]. This exercise shows that many scenarios that seem interesting in the short term (next 3 months) actually fail to meet demand in the medium term ( 9 to 12 months).

Another crucial issue of our problem is the fact that usually the DES approach produces a graphic display of physical movements of productive system units. Such a spatial representation is possible where operations are performed at different places by different static work centers, to which items are moved to be processed. This is not the case in our simulation problem since the items to be processed (mine 
parcels) cannot be moved and are successively occupied by moving work centers corresponding to dedicated or multipurpose machines (able to perform one to three of the seven basic operations) that extract the ore at a given layer, before moving on to the next ore quality, in another parcel. The mine which we are interested in here (OCP's Benguerir (Morocco) open-pit mine), contains a huge number of parcels among of which only a small subset is targeted for extraction under the planning horizon (in this case five hundred parcels). Thus, the conventional representation would create as many work centers as there are parcels, each successively mobilizing different machines. This complex representation faces yet another problem as items to be processed do not come from a particular entry point but from the parcel itself, through a pile of layer blocks under the parcel surface. In other words, such modelling involves difficult machine management issues and a graphic representation that is little adapted for use in the extraction process.

In light of the above, we chose a different modelling approach, based on logic and a set of parallel processors, each dedicated to a single basic operation and linked by stocks of items (blocks) that are available for a given basic operation and that will move on, once processing has been completed, to the following stock of items waiting for the next basic operation. This modelling choice facilitates simulation monitoring but does away with the dynamic spatial view of the extraction process. Accordingly, in order to obtain a presentation of simulation results, we had to build in their static and dynamic spatial consequences to form a simple, clear and interactive view of complex results. Comparing schedule solutions involves a difficult exploration of all their multidimensional impacts that requires the help of a Decision Support System (DSS) whose original features are presented below.

The scope of our literature review presented under Section II was of course defined by the nature of our scheduling problem as briefly described above. In Section III, we describe the functional and technical bases of the designed interactive scheduling DSS and in Section IV we present an illustration of its use and results. Section V offers a conclusion and discussion for the prospective uses for this DSS).

\section{LITERATURE REVIEW}

In early 1971, the bases of Decision Support Systems (DSS) were laid by [3] before being transformed into a structured approach by [4]. Additionally, information technology (IT) developments greatly augmented DSS potential [5], [6], [7] without altering its rationale. Basically, a DSS can be broken down into: i) an interface formulating a partially structured complex issue in order to define a structured problem; ii) a single or multiple module(s) to solve the structured problem, usually backed by an optimization or simulation model [8]; iii) an interface capable of exploring all the consequences of a given solution as well as of validating or adjusting it; iv) and where no acceptable solution is found and the current formulation has failed to deliver a satisfactory solution, using the feedback information from prior formulations to redefine a new structured problem.
We reviewed a number of papers published in scientific newspapers or proceedings proposing DSS for scheduling problems, our analysis of which is reported in sub-section A. In fact, most Scheduling DSS are based on an optimization model. However, due to the mathematical complexity of our problem, we chose to tackle it differently through a simulation approach, as explained before. Thus, in sub-section B we focus on simulation-based scheduling DSS.

\section{A. Scheduling-oriented DSS}

[9] proposes a DSS framework that aims to schedule the supply chain based on flexible demand, accordingly they chose a model where the decision-making parameters are built into the production management process. These authors propose in [10] the design of a DSS that integrates machine scheduling with inventory management for a multi-product manufacturing industry. They suggest other algorithms in [11] to design a production scheduling model taking constraint parameters into account.

The Web-based Visual DSS developed by [12] aims to schedule the letter delivery process to customers while taking process production planning into account. In order to ensure a high level of customer service quality, the optimal solution is obtained only provided information from different systems is collected and analyzed. One of the strengths of this DSS is the choice of web-based applications which is a reliable advanced technology to model a number of services. However, the scheduling process seems inefficient.

IBM's DSS for paper production scheduling (A-Team) in [13] is made up of three types of agents which work asynchronously: 'constructors', 'improvers', and 'destroyers'. The 'Constructor agent's task is to define the problem and create new solutions. The 'Improver' agent's is to improve the set of solutions by modifying and combining the existing solutions, the 'Destroyer' agent's is to eliminate poor solutions and focusing the effort of the 'Improvers' in order to limit the number of solutions. The Dispatcher is a scheduling decision-support system implemented on VBA by [14].

\section{B. Simulation-based Scheduling DSS}

Many DSSs focus on different scheduling problems and among these some are simulation-based. (GeSIM) is a simulation-based scheduling DSS proposed in [15] for customer-driven manufacturing operation planning. The approach follows definite steps: integrating data, feeding the simulation model, showing the results and supporting interactive modifications. For a correctly defined problem and information, the tool requires user interaction before final input into the GeSIM.

[16] presents a simulation-based Decision Support System for logistics management aiming to improve decisions on a tactical and operational level. The DSS helps manage the flow of goods and business transactions between a port and a dry port in a way that is efficient for real-time management of transport systems and reduction of lead time in the port and dry-port area. They also propose a DSS to manage the flow of goods and business transactions between a port and a dry port based on a simulation approach. Finally [17] provides a DSS 
to understand and simulate the structure of urban system freight and to compute some indicators that, compared with target and benchmarking values, allow to identify its level of service.

\section{DSS DESCRIPTION}

Let us start by describing the functional architecture of our designed DSS (sub-section A) and its technical bases (subsection B).

\section{A. DSS Functional Architecture}

The design of our DSS follows a functional breakdown. In fact, starting from a partially structured problem, we define a structured problem to be simulated. The simulation of the structured problem enables an exhaustive reproduction of the extraction process through parameterized priority assignment rules for machine allocation. This is based on a weighting system that takes into account the availability and accessibility of the required layers (for efficiency purposes) as well as equipment allocation and location (for effectiveness purposes). If the resulting scenario is not feasible, one returns to structured problem formulation and parameterization.

The flowchart in "Fig. 1" matches the original pattern of the DSS designers, with its four components.

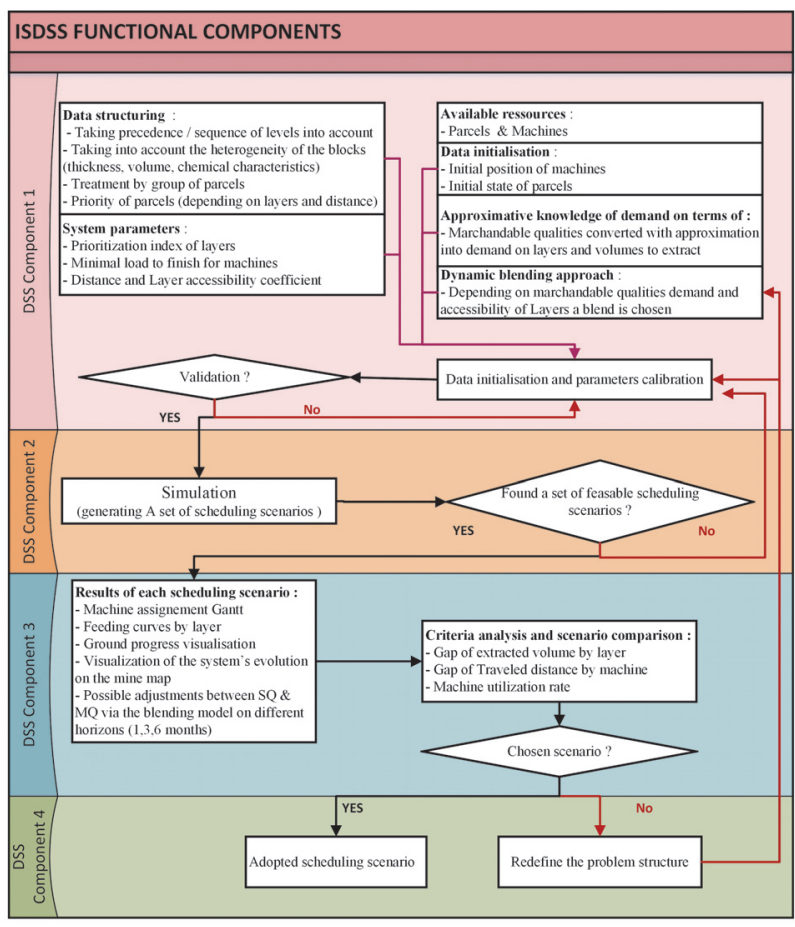

Fig. 1. DSS Functional components

\section{B. DSS Technical Architecture}

Our DSS is based on a Model-View-Control (MVC) architecture with typical DSS functionalities, such as data management, model management and graphic user interface. The DSS is a Java-based web application integrating different
APIs such as leafJS for map presentation (open-source JavaScript library for mobile-friendly interactive maps), JChart (Java Chart) for solution display and Simul8 library to launch the simulation model (it being the software used by the DSS) and also Excel, whose Solver is used to solve the set of LP blending problems to make sure the extracted SQ will meet MQ demand at different planning horizons. The display module is based on Ajax (Asynchronous JavaScript and XML) to retain all of the display functionalities in the browser area without having to request the server for a new page.

As shown in "Fig. 2", our proposed ore extraction DSS for phosphate mine scheduling consists in three key stages.

The first stage concerns business-related data management. There are two kinds of data: structural and dynamic. The structural data reflect field structure such as the layers making up each panel, trenches and parcels. It includes geographical corrodes, machine specifications related to the nature of basic operations that can be handled by each machine as well as the nature of the layer to be extracted by it and its characteristics. The dynamic data reflect the state of the mine: stocks, machine initial position, parcel initial state and progress, etc. Data management is connected to a relational database that stores both kinds of data.

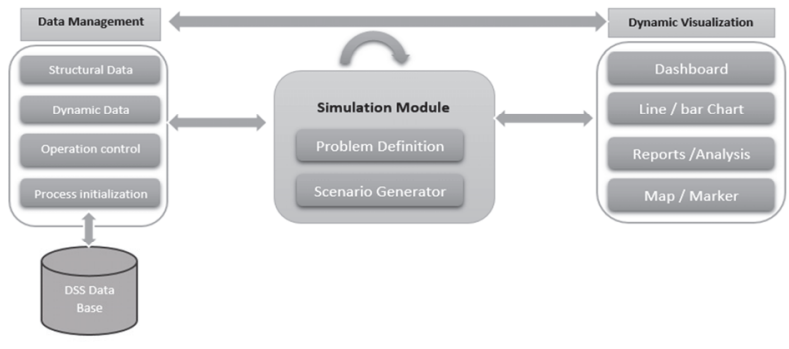

Fig. 2. DSS Architecture

The second stage is a Discrete Event Simulation that serves to model the extraction process using both the structural and dynamic data. It simulates the system and closely tracks progress throughout the extraction process as well as equipment allocation. Finally, the Excel LP solver is used to test the possible adjustments to SQs and MQs based on LP blending options.

The third stage is data display based on the scenario proposed on the simulation stage. The DSS allows users to display a scenario's results using different kinds of formats such as charts, dashboards, tables and map localizations. This stage also offers a functionality to compare different scenarios. If the results are satisfactory, then the decision is submitted to the database by means of the user interface, otherwise a new decision is evaluated.

\section{DSS: IMPLEMENTATION \& EXPERIMENT}

Our developed DSS imports its data from a "Parcels listing"- an Excel file containing information about each parcel, panel, layer etc. This is used to gather the structural data and initialize the DSS to run the simulation using other 
dynamic data. Different datasets were used to evaluate our proposed approach and architecture. In this section, we present an example of a data set and two different scenarios generated with parameter settings produced by the DSS.

The following case study concerns ore extraction scheduling at one of OCP's open-pit mines in Morocco. The initial DSS setup consists in integrating the structural data related to panels, parcels, machines etc. This is followed by parameter setting including layer priority indexes, distances and accessibility coefficients and the minimal equipment load. The simulation model embedded in the DSS then generates different scenarios, and a comparison is made on the bases of such criteria as the volume extracted per layer.

Let us turn to our DSS key component functionalities. In order to ensure an efficient use of the platform, we defined two user profiles: the 'expert' profile is used for simulation scenario parameters to be applied to the various models while the 'read' profile serves to view the collected data and the results of the forecast procedure produced by the modelling system.

In the following section, we simply present a case study for the data display function of the DSS. For this experiment, we used actual data inputs used to run our DSS and to evaluate the solution obtained as further explained below.

The inputs used for this example relate to five panels containing twenty four trenches and five hundred parcels targeted for extraction. In all, fifty machines are allocated to perform the seven basic extraction operations, each with its own specifications and nature, namely multipurpose machine or dedicated equipment.

The simulation outputs are hourly forecasts of feedings for the 10 different ore layers. The hourly grade requirements depend on blended ore (outputs) scheduled production obtained by blending the extracted ore layers (inputs). These blends are dynamically defined and depend on the extracted layers inventories, feedings and also on production schedules. These outputs need to be validated by the blending test which ensures that all the resulting scenarios are valid (meet global demand). The difference between the proposed scenarios boils down to their efficiency and effectiveness, namely the choice of parcels to be extracted and equipment allocation.

Therefore, the acceptability and choice of a simulation solution for this scheduling problem, obtained through particular parameter settings, results from its technical coherence, the ability to meet global demand on the relevant horizon and its performance versus alternative solutions that also meet the first two conditions.

\section{A. Data management}

Our framework applies a relational database scheme based on an object-oriented model to structure its implementation. A dedicated user interface is used to enter the data to ensure structural integrity.

The diversity of the parcels is such that it complicates extraction scheduling immensely. Accordingly, it was necessary to define parcel groups so as to improve the structure of actual mine imported data. The groups were designed with respect to geological characteristics (the composition of each parcel, layer volume and thickness, etc.) These geological characteristics vary a lot from panel to panel, trench to trench and even parcel to parcel. Thus, this grouping approach involves the creation of two new categories: i) the 'sub-panel' (defined as a grouping of parcels that have the same structure, i.e. the same layers and accumulation pattern). ii) a 'group' defined as cases belonging to the same sub-panel containing layers of similar thickness.

Once the structural data is ready for processing, we can initialize the system for launch, using the dynamic data. This data mainly plots initial equipment location, initial stock state as well as initial deposit state. Due to the large volume of data, we designed a tool to import the data from an Excel file supplied by the mine manager. After all the necessary data is entered or imported, the next step is to set the simulation parameters through the problem definition interface.

\section{B. The Discrete Event Simulation Model}

Based on a defined set of parameters, the simulation is run and a set of scenarios is generated. Each scenario represents a scheduling plan including the choice of parcels to be extracted and the machines to be allocated. Thus, for a given scenario, where a proposed result is unfeasible and/or unsatisfactory in terms of efficiency or effectiveness, the system is able to redefine the problem or to create a new one with the aim of achieving a satisfactory scenario to be adopted as a schedule.

\section{Data Display}

In this sub-section, we present two aspects of display of data scenario results and of the comparison between two scenarios.

\section{1) Results of a scheduling scenario}

The DSS presents the scenario evaluation using 4 major tabs. This can be filtered by period range.

The first tab shows the cumulative layer feeding curves as illustrated on "Fig. 3". It presents layer the aggregate extracted by volume by period and allows users to evaluate layer production based on the priority index. It evaluates the proposed scenario by checking whether it meets demand and reports any over- production or under-production situations. The second tab focuses on volume extracted from each layer over a specific period. This chart enables users to view detailed data and extraction progress.

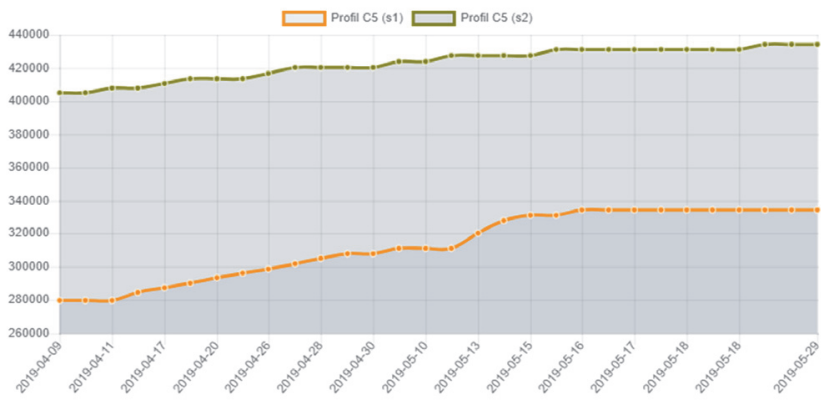

Fig. 3. Cumulative profil layer Feed Curves 
On tab 3 the proposed machine allocation by basic operation is pictured by means of machine Gantts as illustrated in "Fig. 4". For each machine Gantt, the user can view the proposed allocations for it along with timing, nature of the basic operation performed and indication of the parcel, trench and panel the machine is working on at different times. In addition, the user may filter on basic operations or specific machines in order to assess machine performance in terms of occupation and displacement time.

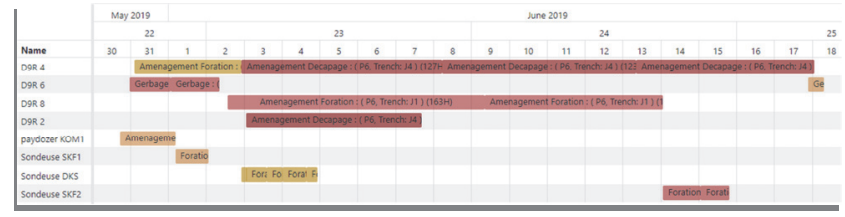

Fig. 4. Machine Gantt

The graphs presented above in "Fig. 3" and "Fig. 4" are used by operations managers to assess scenario short-term and long-term impact and discard any solution that may be relevant in the short-term but counter-productive in the long term.

Finally, for an efficient evaluation of work in the field, the last tab plots the solution on an actual map using mapping technologies to show the different parcels being processed "Fig. 5". The representation uses two different colors: red for parcels in progress and green for extracted parcels. The user can zoom in for more details on the machine, the basic operation it performs or parcel information (thickness, trench and panel) "Fig. 6". In other words, this interface gives a concrete view to support visual scenario validation. The views illustrated in "Fig. 5" and "Fig. 6" can be refreshed every 2 seconds, each step corresponding to mine state 12 hours later, reflecting extraction progress.

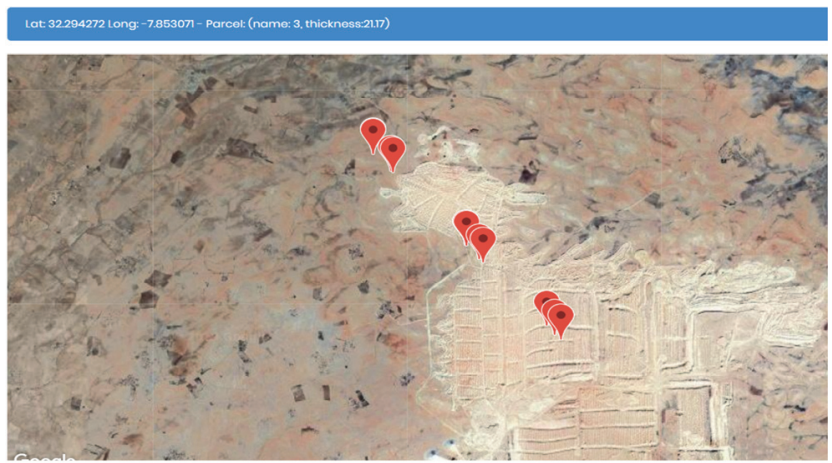

Fig. 5. Parcel and machine mapping

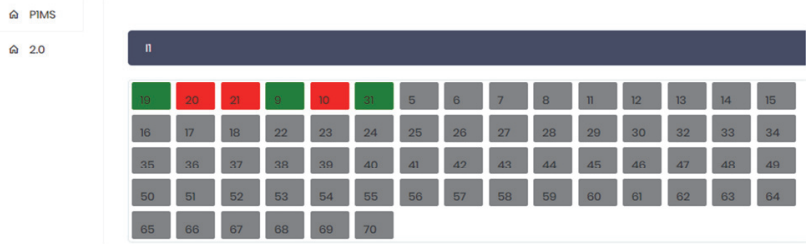

Fig. 6. Detail information of panel processed by trench and parcel

\section{2) Comparative analysis of two different scenarios}

In this case study, two different extraction scenarios based on the defined layer priority index were used. Both had the same structural/dynamic data and were run on the same planning horizon. We present below the performance evaluation graphics for each scenario "Fig. 7".

The feeding curves clearly show the influence of the layer priority index parameter defined separately for each scenario. This index produces differences in terms of extracted volume from each layer and their aggregates. The index also impacts processed parcel choice so as to exploit the layers that are most needed over a determined horizon. This justifies the choice of this variable as a parameter that can be adjusted in the simulation model settings as implemented in our DSS. Accordingly, the model uses the priority index as a primary criterion in producing extraction schedules where layers are arranged in decreasing order of priority from the larger index to the lowest one. According to the state of the mine at system initialization, this may result in extraction from other layers depending on the degree of urgency to meet demand.

Based on results, scenario comparison of the volumes extracted from each layer is possible through the graphic interface by choosing the layer used for comparison purposes "Fig. 7". There is also the option of accurate comparison using the calculated layer gap which is the difference between the cumulative volume extracted for a specific layer in scenario 1 and scenario 2 . The layer gaps serve to facilitate the choice of the most efficient scenario from the set of scenarios that meet the condition of satisfying global demand. In short, these functionalities offer operational managers a chance to form a clear picture of the relevance of each scenario in the planning horizon and for the work field, based on the extracted layers and their volumes in each scenario.

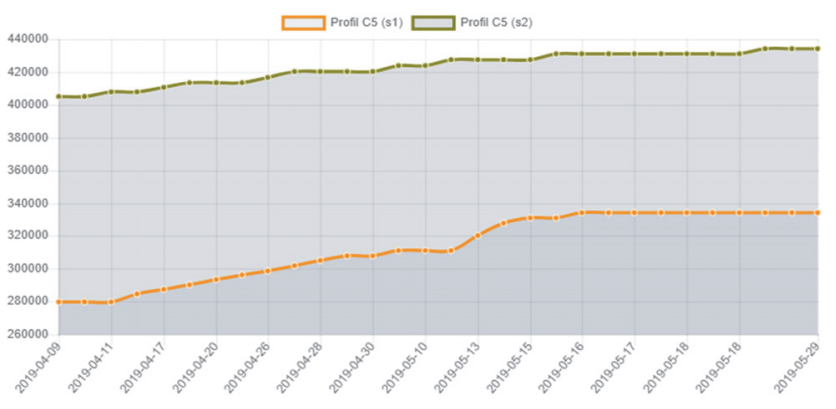

Fig. 7. Two scenarios aggregate feeding curves juxtaposed by profil layer

Additionally, the operational manager may compare the scenarios under review in terms of machine occupation and displacement time by period. As already noted, each task describes the allocation of a machine to one or several basic operations depending on its nature machine (dedicated or multi-purpose).

In this case too, results of the comparison can be plotted graphically as in "Fig. 8", which shows the different occupation and displacement time for each machine. Again, the manager may obtain an accurate calculation of the machine occupation gap between two scenarios. In the same 
way, displacement time machine gap between two scenarios may be calculated. These different results provide a reliable picture of the frequency of displacement and occupation time per machine for the two compared scenarios, which greatly facilitates the selection of an adequate scenario in terms of both efficiency and effectiveness. The operational manager can choose the scenario in light of the juxtaposed machine Gantts and the occupation and displacement time chart for each machine and the calculated gaps.

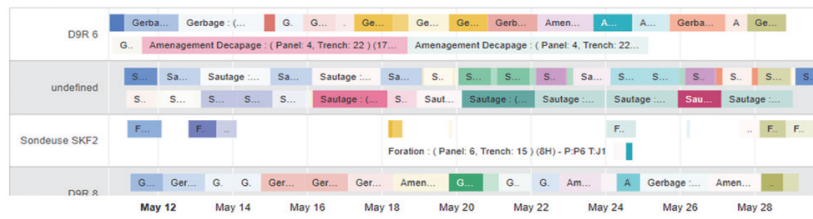

Fig. 8. Machine Gantts juxtaposed for two scenarios

Indeed, through a precise evaluation of one or multiple scenarios, our DSS helps the decision-maker to quantify the impact and choose the best solution very quickly. Technically, the DSS generates a retroactive loop that helps define/redefine the problem in order to obtain a different set of solutions to be analyzed and compared to others. Accordingly, the decisionmaker will then either select an adequate scenario or return to the parameterization interface

\section{CONCLUSION \& PROSPECTS}

This paper discusses the design of an Interactive Scheduling DSS based on Discrete Event Simulation which helps the user to select the best scheduling scenario among the set of feasible ore extraction scenarios capable of meeting demand in merchantable qualities in an efficient and effective way. The simulation model reflects the complex extraction process through parameterized priority assignment rules for the allocation of machines according to a weighting system that takes into account multiple constraints including the availability and accessibility of the targeted layer and equipment availability and location. The DSS is currently being tested with real life data with different parameters in order to fine-tune its design for optimal relevance and robustness.

Our vision aims to widen the decision-making horizon from a few weeks (short term) to a one-year horizon (medium term) through a number of adjustments to the simulation model. In particular, the coupling of the scheduling simulation model and the blending optimization model offers attractive prospects. This will allow the users to obtain different scenarios focused simultaneously on efficient and effective scheduling as well as blending feasibility. Ultimately, therefore, we aim to improve the overall consistency of the decisions made locally by different supply chain actors as well as achieve a close and flexible match of output with known and forecasted demand underpinned by a well-designed DSS approach.

\section{References}

[1] A. Azzamouri et V. Giard, "Dynamic blending as a source of flexibility and efficiency in managing a phosphate supply chain," ILS Conference, July 8-11, Lyon, France p. 12, 2018.

[2] Azzamouri, A., Essadi, I., Fénies, P., Fontane, F, Giard, V., "Interactive Scheduling Decision Support System: case study for fertilizer production on supply chain," ICTO, Information and Communication Technologies in Organizations and Society, Springer, 2017.

[3] Gorry, G., Morton, M., "A framework for management information systems",Sloan Management Review, p. 49-61,Spring, 1989.

[4] Keen, P., Scott Morton, M., "Decision Support Systems: An Organizational Perspective,". Addison-Wesley Publishing, Reading, 1978.

[5] J. P. Shim, M. Warkentin, J. F. Courtney, D. J. Power, R. Sharda, et C. Carlsson, "Past, present, and future of decision support technology," Decision Support Systems, vol. 33, n 2, p. 111-126, juin 2002.

[6] S. Eom et E. Kim, "A survey of decision support system applications (1995-2001)," Journal of the Operational Research Society, vol. 57, $\mathrm{n}^{\circ}$ 11, p. 1264-1278, nov. 2006.

[7] H. K. Bhargava, D. J. Power, et D. Sun, "Progress in Web-based decision support technologies," Decision Support Systems, vol. 43, $\mathrm{n}^{\circ} 4$, p. 1083-1095, august 2007 .

[8] D. J. Power et R. Sharda, "Model-driven decision support systems: Concepts and research directions," Decision Support Systems, vol. 43, no 3, p. 1044-1061, avr. 2007.

[9] F. Galasso , C. Mercé \& B. Grabot, Original Articles, "Decision support framework for supply chain planning with flexible demand,", International Journal of Production Research, vol. 47, p. 455-478,2009.

[10] Krishna, L., Janardhan, G., Rao, C, "Web Integrated Decision Support System for Machine Scheduling and Inventory Management," IUP Journal of Operations Management, vol. 8, p. 35-52, Feb 2009.

[11] Tang, L., Liu, G, "A mathematical programming model and solution for scheduling production orders in Shanghai Baoshan Iron and Steel Complex," European Journal of Operational Research, vol. 182, p. 14531468, nov. 2007.

[12] K. Krishnaiyer et F. F. Chen, "Web-based Visual Decision Support System (WVDSS) for letter shop," Robotics and Computer-Integrated Manufacturing, vol. 43, p. 148-154, févr. 2017.

[13] P. Keskinocak et al., "Scheduling Solutions for the Paper Industry," Operations Research, vol. 50, p. 249-259, avr. 2002.

[14] T. Freed, K. H. Doerr, et T. Chang, "In-house development of scheduling decision support systems: case study for scheduling semiconductor device test operations," International Journal of Production Research, vol. 45, no 21, p. 5075-5093, nov. 2007.

[15] Heilala, J., Montonen, J., Jarvinen, P., Kivikunnas, S., Maantila, M., Sillanpaa, J., Jokinen, T., "Developing simulation-based Decision Support Systems for customer-driven manufacturing operation planning," in: Proceedings of the 2010 Winter Simulation Conference, 2010.

[16] M. P. Fanti, G. Iacobellis, W. Ukovich, V. Boschian, G. Georgoulas, et C. Stylios, "A simulation based Decision Support System for logistics management," Journal of Computational Science,2014.

[17] A. Comi et L. Rosati, "CLASS: A DSS for the Analysis and the Simulation of Urban Freight Systems," Transportation Research Procedia, vol. 5, p. 132-144, 2015.

[18] R. N. Sharma, N. Chand, V. Sharma, et D. Yadav, "Decision support system for operation, scheduling and optimization of hydro power plant in Jammu and Kashmir region," Renewable and Sustainable Energy Reviews, vol. C, no 43, p. 1099-1113, 2015.

[19] L. F. R. Camargo, L. H. Rodrigues, D. P. Lacerda, et F. S. Piran, "A method for integrated process simulation in the mining industry," European Journal of Operational Research, vol. 264, no 3, p. 1116-1129, févr.2018 